\title{
Refronterización de la migración en tránsito por México. El caso de las migraciones centroamericanas por la Zona Metropolitana de Guadalajara
}

\author{
Rebordering of migration in transit through Mexico. \\ The case of Central American migrations through the \\ Metropolitan Area of Guadalajara
}

Recibido el 29 de septiembre de 2018. Aceptado el 18 de septiembre de 2019. Publicado el 9 de octubre de 2019.

*Autor para correspondencia: Rafael Alonso Hernández López, correo electrónico: rahernandez@ colef.mx

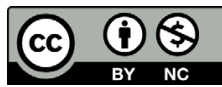

Esta obra está protegida bajo una Licencia Creative Commons Atribución-NoComercial 4.0 Internacional.

\author{
Rafael Alonso Hernández López a* (DD https://orcid.org/0000-0002-1233-9242 \\ Iván Francisco Porraz Gómez ${ }^{\text {b }}$ (i) https://orcid.org/0000-0002-6424-5416 \\ Mariana Morante Aguirre ${ }^{c}$ (D) https://orcid.org/0000-0001-7321-629X
}

\begin{abstract}
a El Colegio de la Frontera Norte, México, correo electrónico: rahernandez@colef.mx
${ }^{\text {b }}$ El Colegio de la Frontera Sur, México, correo electrónico: iporraz@ecosur.mx c Dignidad y Justicia en el Camino. A.C., México, correo electrónico: marianamoranteaguirre@ gmail.com
\end{abstract}

\section{Resumen}

El objetivo del presente artículo es analizar cómo la creciente securitización y militarización de las políticas migratorias mexicanas en contra de la migración irregular ha dado pie a recientes refronterizaciones marcadas por el ensanchamiento y multiplicación de las fronteras a lo largo del territorio nacional, y los intentos -desarticulados- de gubernamentalización de las migraciones en tránsito. Para lograr dicho análisis nos enfocamos en regiones tradicionalmente no consideradas zonas fronterizas, en ese sentido buscamos una interpretación de las políticas espaciales (fronteras físicas y simbólicas) en la Zona Metropolitana de Guadalajara, México. En el texto se discute el carácter espacial del poder, evidenciando los mecanismos que transforman a determinados sujetos en cuerpos autorizados y no autorizados para transitar, permanecer o ser reconocidos.

Palabras clave: Refronterización, migración en tránsito, centroamericanos, gubernamentalidad.

\section{Abstract}

The objective of this article is to analyze how the increasing securitization and militarization of Mexican migration policies against irregular migration has led to recentrefronterizations marked by the widening and multiplication of borders throughout the national territory, and attempts - disjointed- of governmentalization of migrations in transit. To achieve this analysis we focus on regions traditionally not

CÓMO CITAR: Hernández, R. A., Porraz, I. F. y Morante, M. (2019). Refronterización de la migración en tránsito por México. El caso de las migraciones centroamericanas por la Zona Metropolitana de Guadalajara. [Rebordering of migration in transit through Mexico. The case of Central American migrations through the Metropolitan Area of Guadalajara]. Estudios Fronterizos, 20, e032. doi:https:// doi.org/10.21670/ref.1911032 
considered border areas, in that sense we seek an interpretation of space policies (physical and symbolic boundaries) in the Metropolitan Zone of Guadalajara, Mexico. We discuss the spatial nature of power, demonstrating the mechanisms that transform certain subjects into authorized and unauthorized bodies to transit, remain or be recognized.

Keywords: Rebordering, migration in transit, Central Americans, governmentality.

\section{Introducción}

La creciente securitización y militarización de las políticas migratorias mexicanas en contra de la migración irregular ha sido ampliamente documentada tanto por académicos como Fassin (2011), Heyman y Ackleson (2009) y Casillas (2008), como por organizaciones de la sociedad civil (REDODEM, 2015). Por otro lado, los instrumentos institucionales para fortalecer la seguridad en el área (México-Centroamérica) han sido varios: el Grupo de Alto Nivel de Seguridad México-Guatemala (Ganseg) y el Grupo de Alto Nivel de Seguridad Fronteriza México-Belice (GANSEF). En el marco de las relaciones con América del Norte, la Alianza para la Seguridad y Prosperidad de América del Norte (ASPAN), y con Estados Unidos, la Iniciativa Mérida cuyo objetivo es la "cooperación en materia de seguridad regional, el fortalecimiento de la seguridad en la frontera de los tres países, y la construcción de un modelo de comunidad económica fuerte" (García y Villafuerte, 2014), más recientemente, la implementación del Programa Integral Frontera Sur ${ }^{1}$ han no solo incrementado la vigilancia de la frontera sur de México, ${ }^{2}$ sino que han provocado la ampliación de la misma, exponiendo a las personas migrantes en tránsito a una mayor violencia y obligándolos a buscar nuevas rutas y medios de transporte. Estas recientes refronterizaciones han estado marcadas por el ensanchamiento y multiplicación de las fronteras a lo largo del territorio nacional, y los intentos de gubernamentalización de las migraciones en tránsito.

En los lugares de tránsito aparentemente alejados de la frontera sur, tales como la Zona Metropolitana de Guadalajara (ZMG), las refronterizaciones han devenido en un contexto local, ya de por sí complejo en el que la presencia del Estado no es estática o coherente, y donde distintas concepciones acerca de la seguridad y la ciudadanía están siendo constantemente redefinidas y/o reconfiguradas. Las acciones de las autoridades federales, estatales y locales en la zMG parten de imaginarios sociales, políticos y económicos que operan tanto a nivel global como local. En ese sentido, el enfocarse en regiones no tradicionalmente consideradas zonas fronterizas, resulta vital no solo para entender el carácter espacial del poder, y de los procesos estatales, sino también para desmantelar los mecanismos que transforman a determinados sujetos en cuerpos autorizados y no autorizados.

\footnotetext{
${ }^{1}$ Las acciones del Programa Integral Frontera Sur estarían encaminadas a garantizar la seguridad de la región sur del país, de las vías del tren y de los migrantes, mismas que se enmarcan en tres objetivos: a) evitar que los migrantes se pongan en riesgo al usar el tren de carga, $b$ ) el desarrollo de estrategias para garantizar la seguridad y la protección de los migrantes y $c$ ) combatir y erradicar a los grupos criminales que asedian y vulneran los derechos de los migrantes.

${ }^{2}$ Está integrada por los estados de Chiapas, Tabasco, Campeche y Quintana Roo, los cuales limitan geográficamente con Guatemala y Belice, a lo largo de 1149 kilómetros (Encuesta sobre Migración [EMIF SUR], 2012).
} 
El objetivo del presente artículo es brindar una interpretación de las políticas espaciales en la Zona Metropolitana de Guadalajara, México. Se trata de una mirada, basada fundamentalmente en las aportaciones teóricas de Brenner (2004), Massey (2005), Foucault ([1976] 2003) y Chatterjee (2004) a una compleja realidad en donde lógicas económicas, políticas y estructuras administrativas se entretejen para invisibilizar o marginalizar a los migrantes en tránsito por la ZMG.

Desde esta perspectiva, la descripción aquí utilizada se sustenta en 19554 entrevistas realizadas por FM4 Paso Libre ${ }^{3}$ a migrantes en tránsito por la ZMG, durante un periodo que va de mayo de 2010 a mayo de 2015 , se presentan y detallan las particularidades de los flujos migrantes que hacen del corredor ferroviario de occidente un espacio de tránsito migratorio. Además, durante el verano de 2014, se realizaron entrevistas con los alcaldes y funcionarios de Guadalajara y Zapopan, ambos municipios de la ZMG, así como diversos recorridos a lo largo de las vías del tren en los que se documentó tanto el espacio físico, como social que rodea a las vías del tren.

El texto se divide en tres secciones. En la primera se describen las características actuales y dinámicas que configuran la ruta de occidente, en donde Dignidad y Justicia en el Camino, A.C., conocida públicamente como FM4 Paso Libre, brinda atención humanitaria integral a migrantes en tránsito por la zMG. La segunda sección, aborda las tendencias de las refronterizaciones a través de procesos tales como: la ampliación y multiplicación de las fronteras y la (in)gubernamentalidad de los migrantes en tránsito. El tercer apartado esboza algunas conclusiones y recomendaciones para abonar a la construcción de un paso digno y justo para las personas migrantes.

\section{Migración en tránsito por el corredor ferroviario de occidente}

FM4 Paso Libre es una organización de la sociedad civil que desde hace casi diez años realiza un esfuerzo en favor de la población migrante en tránsito y refugiada en el corredor ferroviario del occidente mexicano. La organización en la que actualmente participa un grupo de más de 120 personas, en su mayoría jóvenes estudiantes, realiza su trabajo a través de cinco áreas: $a$ ) Acompañamiento integral (encargada de la atención a las necesidades relativas a la salud física, mental y de inserción social, laboral y educativa para personas que derivado de diversos factores se establecen en la ciudad); b) Vinculación (incidencia en políticas públicas locales, estatales y nacionales, así como el trabajo en redes; incidencia social para sensibilizar a la población local en torno al fenómeno migratorio); c) Atención jurídica (gestión de trámites migratorios, denuncia de violaciones a derechos humanos); $d$ ) Desarrollo institucional (generación de proyectos, procuración, administración y rendición de cuentas de proyectos); e) Investigación (sistematización, análisis, discusión y difusión de la problemática observada en el acompañamiento cotidiano a personas migrantes y refugiadas). Todo ello tiene lugar en el Centro de Atención a Migrantes y Refugiados, espacio que brinda ayuda humanitaria de manera gratuita (alimentación, ropa, insumos para higiene personal, servicios sanitarios, albergue para 100 personas, llamadas telefónicas), la cual ha alcanzado ya más 35000 personas en los últimos ocho años.

${ }^{3}$ La sistematización de este material se encuentra registrado y publicado en el trabajo de FM4 Paso Libre (2013). 
Es precisamente desde esta experiencia de acompañamiento que es posible identificar los cambios en las dinámicas, composición de flujos derivados de las políticas migratorias, tanto externas, particularmente de Estados Unidos, como internas, las cuales han adquirido, como ya se mencionó una perspectiva de securitización y criminalización de las poblaciones migrantes en tránsito por nuestro país.

Para entender la complejidad de esta situación es pertinente ubicarnos espacialmente para sopesar los impactos de políticas gubernamentales que si bien tendrían un componente de acción ubicado en los límites geográficos nacionales, se expanden para reconfigurar por lo menos simbólicamente las fronteras nacionales. De ahí que nuestro análisis se centre en lo que denominamos el corredor ferroviario de occidente.

El referido corredor inicia en la bifurcación ferroviaria de la ciudad de Irapuato, Guanajuato. Continúa su trayecto transitando por importantes ciudades del occidente mexicano, tales como Guadalajara, Tepic, Mazatlán, Culiacán, Hermosillo, Nogales, Mexicali y Tijuana (Figura 1).

Figura 1: Corredores ferroviarios a partir del centro del país

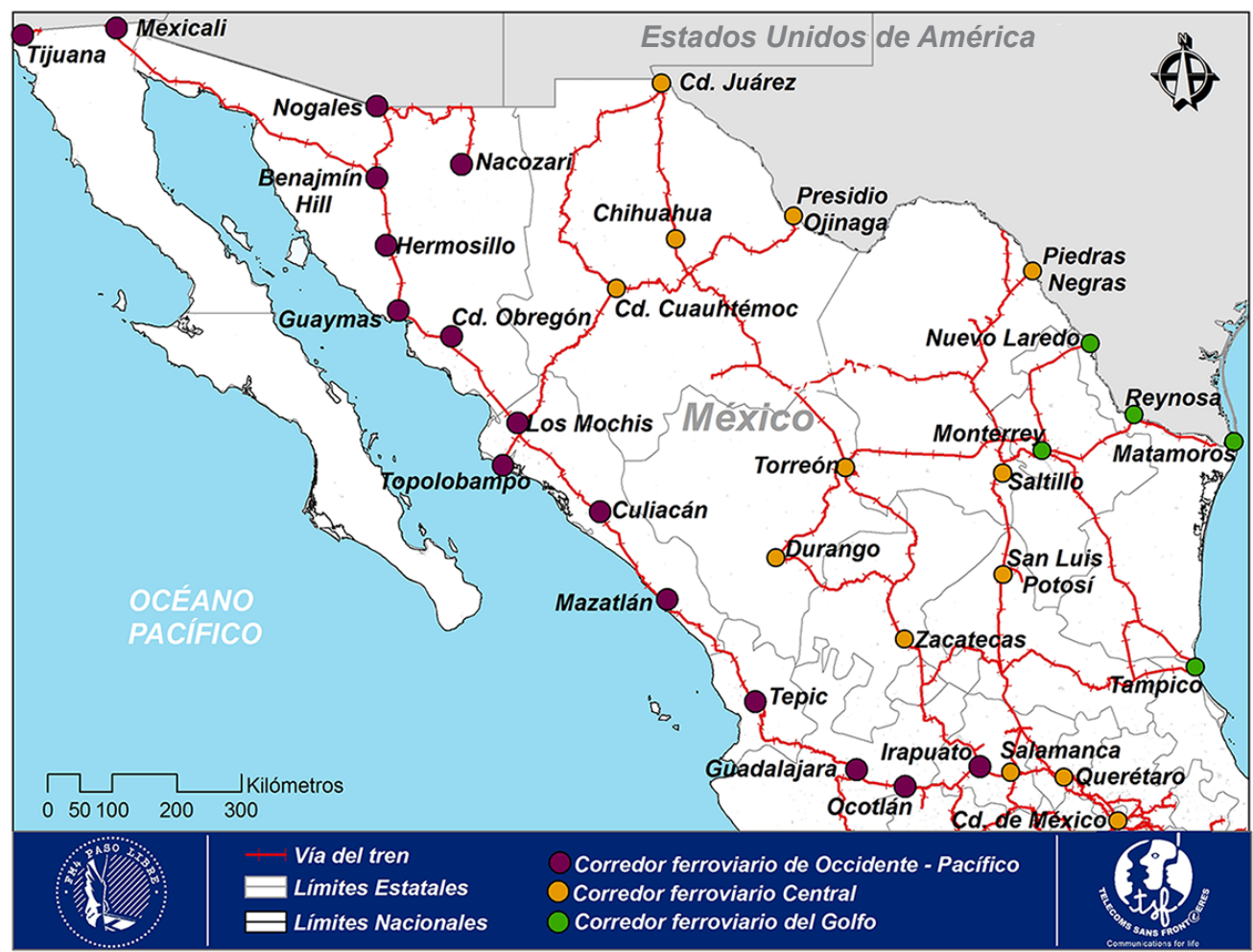

Fuente: Fm4 Paso Libre y Télécoms Sans Frontières, 2014.

Esta ruta es la opción que ofrece mayores garantías en materia de seguridad. No es que la misma se encuentre exenta de agresiones, robos, extorsiones y otras violaciones a derechos humanos, sino porque las personas migrantes realizan, en la mayoría de casos, un cálculo del costo-beneficio antes de utilizarla. 
La ruta de occidente era hasta por lo menos 2010 poco conocida mediáticamente, y poco transitada y concurrida por los migrantes. Es una ruta que recorre alrededor de 4137 kilómetros $^{4}$ hasta Mexicali, duplicando la cantidad de kilómetros de las rutas ferroviaria del centro y del golfo. Transitar por la ruta ferroviaria más larga del país, conlleva varios costos adicionales para las personas migrantes. Aunado al tema de la distancia se encuentra el hecho de que esta ruta ferroviaria atraviesa gran parte del desierto de Sonora, que tiene uno de los climas más hostiles del continente.

La ruta de occidente comenzó a ganar importancia a partir de las diferentes tragedias acaecidas a migrantes en los otros corredores ferroviarios. ${ }^{5}$ Frente a este escenario de violencia los migrantes, de manera cada vez más consciente, optaron por usar la ruta de occidente que aunque más larga y con menos ayuda humanitaria significaba una posibilidad de continuar el tránsito migratorio con vida.

De acuerdo con información documentada por FM4 Paso Libre (2013), ${ }^{6}$ poco más del $50 \%$ de los migrantes que utilizan este corredor por cuestiones de seguridad, argumentan que de esta forma evitan las rutas del golfo y del centro que se caracterizan por su peligrosidad al estar ocupadas por el crimen organizado. Es necesario precisar que esta decisión no solo se basa en lo que sucede con la delincuencia organizada, sino con la violencia ejercida por las autoridades: los robos y la fuerza coercitiva del poder judicial, que se manifiesta en las policías municipales, estatales, federales y las autoridades migratorias.

Dentro de la ruta del Pacífico, Guadalajara se constituye como una de las ciudades más importantes del occidente y del país, y como una ciudad por donde hay un importante tránsito de personas y mercancías. Guadalajara es también epicentro del proyecto de ayuda humanitaria integral para migrantes en tránsito de FM4 Paso Libre, a través del cual, desde 2010, se ha podido dar seguimiento, registrar y documentar el tránsito migratorio de mexicanos y centroamericanos que a continuación se detalla.

Lo primero que hay que decir es que, pese a la creciente feminización de las migraciones en el mundo, y en particular en México (Acharya 2010; De Leon 2013; Toney, 2009), esta se trata de una ruta eminentemente varonil, en donde existe una representación de $96.72 \%$ para los varones frente $4.18 \%$ de mujeres. Si bien estos datos reflejan únicamente las personas que pasan por el centro de atención, y no la totalidad del flujo, FM4 Paso Libre ha calculado que por el centro de atención transitan $70 \%$ de las personas migrantes viajando en la ruta. Los factores que hacen de esta ruta una varonil necesitan investigarse con mayor detenimiento, sin embargo, es posible que se deba a que la ruta de occidente es mucho más larga, y con menos albergues y redes de apoyo.

Otra consideración importante es que se trata de una ruta transitada por adultos jóvenes, en una edad promedio de 31 años. Contrario a las tradicionales explicaciones sobre la migración en tránsito por México, el corredor ferroviario de occidente es escenario de migraciones mixtas, en las que conviven personas extranjeras y nacionales, que van al norte, al sur o incluso en travesía tratando de encontrar puntos propicios para lograr el cruce e internamiento a Estados Unidos. De manera particular, la

\footnotetext{
${ }^{4}$ Tomando como punto de partida el sur del país, concretamente la ciudad de Tapachula, Chiapas.

${ }^{5}$ En agosto de 2010, el caso de 72 migrantes secuestrados, torturados, mutilados, muertos, sin documentos e irreconocibles. En abril de 2011, se encontraron 193 cuerpos en fosas clandestinas igualmente en el municipio de San Fernando, Tamaulipas. En mayo de 2012, se hallaron 49 torsos abandonados en el municipio de Cadereyta, Nuevo León.

${ }^{6}$ Fuente de información de los datos estadísticos usados en este apartado.
} 
población proveniente de la región centroamericana representa $68 \%$ de la muestra de FM4 Paso Libre, ese porcentaje se forma tan solo con $46 \%$ de los hondureños. Los mexicanos por su parte tienen 30\% de representatividad. El resto lo conforman personas de nacionalidades diversas (Costa Rica, Panamá, Colombia, Haití, Perú, Bolivia, etc.).

\section{El Estado moderno: Procesos de refronterización}

La experiencia de la migración en tránsito por México, nos pone frente a un escenario en el que a través de las experiencias cotidianas de los sujetos antes caracterizados, se vuelve pertinente poner sobre la palestra el papel del Estado en la configuración de varias de las dinámicas del tránsito, así como también en las maneras en las que la ciudadanía reacciona frente a la presencia de las personas migrantes en la ciudad.

En State/Space: A Reader, Brenner, Jessop, Jones y MasLeod (2008) explican que el estudio del Estado westfaliano ha estado marcado por su naturalización. En estas concepciones, se considera al Estado como un contenedor estático en el que se desarrollan los procesos económicos y políticos (Brenner et al., 2008, p. 2). Sin embargo, a partir de los años ochenta, un cambio teórico en el estudio del Estado ha cuestionado la pertinencia de marcos analíticos que lo consideran como una entidad inmutable, coherente y cerrada. En cambio, se ha incrementado una atención crítica sobre las espacialidades cambiantes del poder del Estado y la vida política, así como al desarrollo de nuevas metodologías para su estudio.

De manera similar, en New State Spaces: Urban Governance and the Rescaling of Statehood, Brenner (2004) argumenta que mientras que términos como lo local, lo urbano, y lo regional sirven para separar islas territoriales de relaciones sociales, estos esconden la profunda imbricación mutua que existe entre todas las escalas. Brenner, por ejemplo, explica que las naciones europeas pasan por un proceso de reestructuración espacial. $\mathrm{Y}$ aunque su análisis se centra en una geografía distinta, este aún resulta útil para analizar el caso de la migración en tránsito por México. Para Brenner, el estudio del Estado debe ser aproximado a partir de "procesos continuos que enfatizan las geografías polimorfas en múltiples escalas espaciales" (Brenner, 2004, p. 7) e identifica a la refronterización del Estado como una de las reestructuraciones espaciales más importantes del mismo, y se refiere a un "proceso en el que las fronteras no son vistas exclusivamente como demarcadores de la soberanía, son más bien entendidas como prácticas semióticas, simbólicas, y político-económicas a través de las cuales el poder del Estado es articulado y desafiado" (Brenner, 2004, p. 7).

En ese sentido, la frontera se entiende no como un límite territorial del Estado, sino como las prácticas dirigidas a donde se encuentran las personas migrantes (Burridge et al., 2017). Por estas razones, conceptualizaciones utilizadas frecuentemente para explicar el tránsito de personas migrantes por México como el de la frontera vertical (Casillas 2008; Torre-Cantalapiedra y Yee-Quintero, 2018) resultan insuficientes para capturar la complejidad, ambivalencia y carácter selectivo de los nuevos controles migratorios. Hablar de una frontera vertical -como sucede con las metáforas espaciales relacionadas con las fronteras- tiende a disfrazar, domesticar y homogenizar los espacios, dando una imagen de estabilidad y permanencia (Burridge et at., 2017) que no refleja la realidad de los procesos de refronterización. 
En la ZMG estos procesos de refronterización se han manifestado en la ampliación y multiplicación de las fronteras, en la consolidación de una doble imaginación geográfica, y en los crecientes intentos del Estado por controlar/manejar la migración en tránsito. Estas dinámicas y procesos de refronterización no son por supuesto, consistentes ni coherentes en todos los niveles del Estado, ni en sus instituciones, ni sus actores, y por el contrario, se contradicen y superponen para crear espacios y grietas institucionales que impactan las trayectorias de las personas migrantes en tránsito.

\section{Ampliación y multiplicación de las fronteras}

Desde la experiencia de FM4 Paso Libre en la ZMG, la ampliación y multiplicación de las fronteras se hace manifiesta a través de la implementación de una política nacional que tiene que ver con el forzamiento del vínculo seguridad nacionalmigración, ratificada mediante acuerdos económicos y políticos. Prueba de ello, es el lanzamiento durante el verano del 2014 del denominado Programa Integral Frontera Sur.

Está ampliamente documentado que desde los ataques del 11 de septiembre de 2001, los Estados Unidos incrementaron la vigilancia y militarización de su frontera con México, presionando además a los países del continente para que hicieran lo propio e impidieran el acceso de personas sin documentos a sus respectivas geografías nacionales, so pretexto de la infiltración de terroristas.

Como parte de las estrategias para atacar al crimen organizado, en 2008 se firmó la Iniciativa Mérida, programa que hasta la fecha (con sus respectivas modificaciones) ha servido como un mecanismo de seguridad que considera al tema migratorio como prioritario. Su tercer pilar, "construyendo una frontera para el siglo xxı", incorporó a la frontera sur de México como un área de interés. Con este programa, Estados Unidos ha aportado \$24 millones de dólares en equipo y asistencia técnica, incluyendo kioskos móviles operados por el Instituto Nacional de Migración (INM), equipos caninos y la capacitación de agentes estadounidenses a personal mexicano dedicado a patrullar las fronteras (Ribando y Finklea, 2017, p. 22). Este tipo de acuerdos consolidaron a la migración como un asunto de seguridad nacional, lo que ha desembocado en la criminalización de las personas migrantes en tránsito por nuestro país.

Pese a todas las acciones por contener los flujos migratorios, la política migratoria mexicana se vio fuertemente confrontada en el verano del 2014, cuando se mediatizó la llamada crisis de los menores migrantes. Estados Unidos, hasta julio de ese año, tenía asegurados más de 50000 indocumentados, muchos eran menores no acompañados y en su mayoría centroamericanos. En este contexto, se dio la presentación del Programa Integral Frontera Sur del Estado mexicano el 7 de julio de 2014, cuya finalidad, afirmó el presidente Enrique Peña Nieto consistía en: proteger a los migrantes que cruzan nuestro país y garantizar la seguridad de la región sur del país y de las vías del tren. Ello a través de la implementación de tres objetivos: a) evitar que los migrantes se pongan en riesgo al usar el tren de carga, $b$ ) el desarrollo de estrategias para garantizar la seguridad y la protección de los migrantes y, c) combatir y erradicar a los grupos criminales que asedian y vulneran los derechos de los migrantes.

Estos objetivos irían acompañados, se dijo, de una estrategia de prevención, a través del monitoreo de las vías del tren, garantizando la presencia del Estado 
para evitar delitos, así como el fortalecimiento de la articulación con consulados de los países centroamericanos. En atención a lo anterior, desde junio de 2014 se comenzaron a expedir tarjetas de visitante regional, pero únicamente para ciudadanos guatemaltecos y beliceños; sin considerar, que el mayor flujo migratorio en tránsito en los últimos años ha sido de los hondureños. Se anunció, además, el desarrollo de acciones de inteligencia para desarticular bandas del crimen organizado que dirigen redes de trata, tráfico de personas, extorsión y secuestro hacia migrantes, así como el fortalecimiento de fiscalías de atención a delitos en contra de migrantes, ministerios públicos especializados, la participación de las comisiones estatales de derechos humanos, así como la instalación de albergues operados por la sociedad civil.

Las primeras líneas de acción cubiertas por el Programa Integral Frontera Sur se centraron en la inversión en infraestructura ferroviaria, decantado en medidas de seguridad no para los migrantes, pero sí para los trenes y las mercancías que transportan. Derivado de lo anterior, se han hecho constantes las redadas del INM apoyado por la Policía Federal, el Ejército y la Marina, el resultado: el incremento de la vulnerabilidad, mayor exposición al riesgo, diversificación de rutas, proscribiendo de nuevo a las y los migrantes.

En este nuevo escenario, las redadas contra las y los migrantes se justificaron bajo un discurso ambiguo de defensa y protección de los derechos humanos de los migrantes. El secretario de Gobernación durante el sexenio anterior, Miguel Ángel Osorio Chong, afirmó semanas después del inicio del programa que el plan no era para agredir o lastimar, sino evitar que los migrantes centroamericanos abordaran "la bestia", se buscaba entonces, protegerlos. De hecho y siguiendo la lógica de las autoridades, el éxito del Programa Integral Frontera Sur, se pudo palpar solo unas pocas semanas después de su implementación.

Efectivamente, a través de los constantes operativos implementados en ese contexto el número de migrantes sobre el ferrocarril de carga disminuyó de manera considerable. El asedio por parte de las autoridades migratorias tuvo como escenario no solo las vías del tren, los operativos se expandieron incluso a ciudades y pueblos donde ordinariamente se concentraban los migrantes para esperar movilizarse, además de llevarse a cabo en las inmediaciones de los albergues, comedores y centros de atención humanitaria. Poco a poco los límites de la frontera sur se extendieron hasta prácticamente el centro del país con puntos de verificación migratoria, puestos de inspección militar a lo largo de las rutas carreteras que llegan al centro del país, a fin de detectar a los migrantes que viajan en microbuses, autobuses, coches y tráileres, tal como dan constancia las organizaciones del país que laboran atendiendo a la población migrante en tránsito.

De acuerdo con información de estas organizaciones, el impacto del programa fue tal que en algunos casos la atención que brindaba a los migrantes se redujo hasta en $50 \%$ desde su puesta en marcha. Lo cual no significaba que los migrantes estuvieran dejando de internarse en el país, sino como se mencionó anteriormente, comenzaron a buscar nuevas alternativas, la mayoría de ellas, más riesgosas, en razón de la necesidad de ocultarse de los operativos migratorios. Ahora la población migrante

\footnotetext{
7 "La bestia" es una forma de nombrar al tren de carga en el que se transportan las personas migrantes. Se trata pues de un transporte no ideado para movilizar pasajeros sino mercancías. La analogía con la bestia tiene que ver con lo impactante que es esta maquinaria de acero, en cuyo viaje un descuido puede cobrar la vida de las personas migrantes o la amputación de alguna extremidad.
} 
está improvisando estrategias, caminando por días y semanas en espacios geográficos inhabitados, con climas extremos, en los que además de la vulnerabilidad natural que suponen estos espacios, se potencia la vulnerabilidad por la impunidad con la que operan grupos delictivos que atacan a las y los migrantes.

Si bien es cierto que en Jalisco no existe una estación migratoria de gran capacidad, se cuenta con una en la que permanecen en detención personas que son aseguradas en el estado de Jalisco, así como en algunos puntos cercanos a esta demarcación geográfica. En este sentido se explica el hecho de que los primeros operativos registrados como parte del Programa Frontera Sur se dieran en este estado, a pesar de no contar con la infraestructura y condiciones suficientes para albergar a las personas migrantes. De acuerdo con testimonios de migrantes en tránsito y otras personas habitando en las vías, durante el verano de 2015, el INM comenzó a realizar hasta dos operativos por semana en la zona de las vías del tren.

Los escenarios descritos en esta sección son reflejo de un incremento de los controles migratorios y de la securitización de la política migratoria, que han derivado no solo en la multiplicación de prácticas migratorias anteriormente confinadas a los límites territoriales de México, sino a pasar por un proceso de externalización (Burridge et al., 2017); es decir, por esfuerzos explícitos para involucrar a más instituciones -en este caso el ejército y la Policía Federal-, y por supuesto para extenderse a lo largo del territorio nacional.

\section{La doble imaginación geográfica}

A la ampliación y multiplicación de fronteras, le sigue el carácter selectivo de las fronteras, su función como herramientas para segmentar y diferenciar-no únicamente entre distintas poblaciones-, sino entre los bienes y el capital, y la población migrante en tránsito. Sin embargo, contrario a lo que ocurre con la política nacional de migración, en estos espacios, la autoridad federal es indiferente a la presencia de personas migrantes en la ZMG, excepto cuando representan un obstáculo al libre tránsito de bienes y de capital.

Por ley, la Policía Federal no puede detener a migrantes en tránsito a menos que cometan un delito. Por consiguiente, el papel de la Policía Federal en la zMG en relación con las personas migrantes en tránsito se ha basado en las frecuentes extorsiones y agresiones, y en proteger el libre tránsito de los bienes que son transportados a través de las vías ferroviarias.

En la ZMG, el tren entra por el municipio de El Salto, y hasta Las Juntas en Tlaquepaque, la policía federal baja a los migrantes del tren para que este ingrese a los patios de entrada. Pero a lo largo de ese trayecto se pueden observar, con frecuencia, patrullas de la Policía Federal a las orillas de las vías, escondidas entre los matorrales. Un mayordomo de uno de los trenes que va entrando a la ZMG asegura que a la Policía Federal en realidad no le interesan los migrantes que viajan en el tren: "Bueno... casi no los bajan, sólo cuando la carga del tren es valiosa (...) Ellos tienen que cuidar la "mercancía", los migrantes no les importan, excepto cuando les pueden sacar algo. Los bajan para proteger al tren" (mayordomo, 2014). ${ }^{8}$

${ }^{8}$ El nombre del mayordomo no se revela puesto que el informe consentido que firmó para la entrevista establecía que su identidad se mantendría en anonimato. 
Esta misma lógica con la que opera la Policía Federal es replicada por Ferromex, la empresa dueña de la ruta ferroviaria más grande de México y que cruza por Guadalajara. Esta empresa ha contratado seguridad privada para patrullar las vías del tren a lo largo de su trayecto por México.

En la ZMG, un habitante de la comunidad de El Verde en el municipio de El Salto, por donde entra el tren, afirma:

Los del grupo Eulen pasan seguido por aquí. El otro día, tres migrantes estaban afuera de mi casa esperando a que les trajera agua, y cuando salí ya los estaban molestando, pero como les dije que venían a mi casa se tuvieron que ir (habitante de El verde, 2014).

Ambas instituciones, la Policía Federal y Ferromex configuran un escenario en el que los migrantes son convertidos en una amenaza para los bienes que circulan a través del país. En el sistema capitalista, no hay fronteras para los bienes, estos son transportados a otras partes del país y de Estados Unidos como símbolos del progreso y el crecimiento económico. El movimiento de los migrantes, por otro lado, es visto como una amenaza a la seguridad. Doreen Massey (2005) ha llamado a esta lógica aparentemente contradictoria, una "doble imaginación geográfica" donde uno está marcado por la movilidad y boderlessness mientras el otro está determinado por una férrea disciplina fronteriza. Una doble imaginación que trabaja en favor de los que ya son de por sí poderosos. En el tren de carga, los bienes viajan libremente protegidos por la Policía Federal y por la seguridad privada de Ferromex. La lógica capitalista legitima, respaldada en el Estado, el derecho y poder de las empresas para mover sus bienes de un lugar a otro con relativa facilidad. Sin embargo, el trayecto de los migrantes que viajan en el tren tiene que ser clandestino, y expuesto a mayores peligros sin protección del Estado que en lugar de asumir su responsabilidad de garantizar los derechos humanos y la dignidad de quienes se encuentran en su territorio, los expone a una mayor vulnerabilidad.

\section{La (in)gubernamentalidad de los migrantes en tránsito}

El fortalecimiento de las tendencias de refronterización, se sirve como hemos visto, de acciones y estrategias que suceden en el espacio físico (no circunscrito necesariamente a los límites territoriales de un Estado) pero que no acaban en él, pues tienen un fuerte componente de carácter simbólico que da cuenta, a su vez, de las lógicas en las que el Estado ejerce su poder sobre las personas.

En ese sentido, en Society must be defended: lectures at the Collège de France, 1975-76, Foucault argumenta que durante la segunda mitad del siglo xviII emergió una nueva tecnología regulatoria del poder, ya no dirigida al cuerpo, sino a la raza humana. En ese sentido, la biopolítica trata con la población como un "problema político para quien mecanismos reguladores deben establecerse para imponer un equilibrio" (Foucault, [1976] 2003). La consecuencia directa de este proceso ha sido la gubermentalización del Estado, en donde la relación que se establece entre el gobierno y las poblaciones está basada en políticas asistencialistas, y no en la participación. Como consecuencia, este 
[...] régimen asegura su legitimidad no en la participación de los ciudadanos en los asuntos de Estado, pero en proclamarse como el proveedor de bienestar a la población. Este modo de razonamiento no es de apertura deliberada, sino de una noción instrumental costo-beneficio (Chatterjee, 2004, p. 34).

Siguiendo a Foucault, este Estado es intrínsecamente racista, es decir, un Estado en el que las amenazas, ya sean internas o externas a la población y por la población son creadas y justificadas, y el "imperativo de matar es aceptable sólo si resulta no en la victoria sobre los adversarios políticos, sino en la eliminación de la amenaza biológica" (Foucault, [1976] 2003, p. 256). Esta eliminación, aclara Foucault, no significa solamente "el asesinato como tal, sino el incrementar el riesgo de muerte para ciertos grupos, o simplemente, la muerte política, la expulsión, el rechazo, entre otros". Este es el poder del Estado que puede "hacer vivir, o dejar morir" (Foucault, [1976] 2003, p. 257).

Como resultado, el tipo de relación política que el Estado establece con la población es de una naturaleza diferente a aquella que establece con los ciudadanos. Tal como lo señala Chatterjee (2004), muchos habitantes son "solo ambigua y contextualmente ciudadanos en el sentido imaginado por la Constitución. No son, por tanto, miembros - propios- de la sociedad civil" (Chatterjee, 2004, p. 38). Pero, y en esto Chatterjee es enfático, como poblaciones, tienen que ser controlados y manejadas por las agencias gubernamentales, y la relación que el Estado establece con ellas es siempre contextual y temporal.

Los argumentos de Foucault ([1976] 2003) y Chatterjee's (2004) presentan puntos útiles para el análisis del caso de la migración en tránsito por la zMG. Primero, las autoridades locales, enfrentadas a la presencia de los migrantes en tránsito en la ciudad, se resisten a reconocer el carácter de los sujetos que atraviesan la ciudad como sujetos de derecho y no solo de asistencia. Los migrantes en tránsito viajando en el tren de carga, ya sea con documentos o sin documentos, viajan en "los márgenes" en medios de transporte clandestinos y trayectorias inciertas, y por tanto, resisten y escapan a los esfuerzos de las autoridades por contarlos, categorizarlos, y estabilizarlos en ciertos lugares. Lo que es más, su casi siempre temporal estancia en la ciudad hace su clasificación y control más difícil para las autoridades. Segundo, las autoridades locales niegan cualquier responsabilidad con las vidas de las personas migrantes en tránsito, y el tipo de relaciones que establecen con estas -cuando lo hacen- se basan en políticas asistencialistas frágiles y coyunturales. Tercero, a través de logísticas racistas, como las explicadas por Foucault, las autoridades locales han enmarcado a las personas migrantes en tránsito como una amenaza de salud para los ciudadanos, es decir, como una amenaza biológica.

En entrevistas realizadas con los alcaldes de los municipios de Zapopan y Guadalajara durante el verano de $2014,{ }^{9}$ se pudo identificar que para las autoridades locales el problema de los migrantes en tránsito se encuentra en no poder saber quiénes son, dónde están, de dónde vienen, y dónde van. Cuando al presidente municipal de Zapopan se le preguntó si pensaba que tenía responsabilidad sobre los migrantes en tránsito por su municipio, contestó: "Focalizar la atención al migrante se hace muy

\footnotetext{
${ }^{9}$ El nombre de los alcaldes de los municipios de Zapopan y Guadalajara no son revelados debido a que el informe consentido que firmaron para la entrevista establecía que su nombre no sería utilizado y únicamente se mostraría el periodo de su administración que es 2012-2015.
} 
difícil, en primer lugar porque no sabes quiénes son. Pues sí, son caras pero no son nombres ni son apellidos [...] ni los tienes ubicados físicamente en algún espacio" (alcalde de Zapopan, 2014). En este sentido, desde la perspectiva de la autoridad local, el control y la categorización resultan indispensables para la provisión de servicios.

Dentro de la gubernamentalización del Estado, el ser una cara, un ser humano, no es condición suficiente para acceder a servicios, o incluso ser reconocido como sujeto de derecho porque además, se necesita tener un nombre, una identidad que el Estado pueda comprobar. Durante la misma entrevista, se le preguntó al alcalde su opinión acerca de la opción de establecer una visa de migrante en tránsito para que pudieran cruzar por México. Su respuesta fue:

Eso ayudaría mucho, a contar con un padrón, a tener una visión más clara de dónde están y qué haciendo y a poderlos sumar, o poderlos subir al marco jurídico, al marco legal... además de alguna manera ya cuentas con registro de quiénes son, a dónde van, de cuáles son sus proyectos (alcalde de Zapopan, 2014).

Las personas migrantes en tránsito generan ansiedad entre las autoridades locales porque no pueden ser reconocidas con sus instrumentos estadísticos, necesarios para la provisión de bienestar y servicios, lo que supone, frente a todo el clasificarlos en determinadas categorías y espacios. Justamente en esa dirección, en el año 2016 FM4 Paso Libre en colaboración con el diputado independiente Pedro Kumamoto presentaron al Congreso de Jalisco una iniciativa de ley denominada Ley de Hospitalidad (El Informador, 2016). Dicha iniciativa fue puesta en discusión con otra presentada por la diputada Juana Ceballos del Partido Revolucionario Institucional (PRI), denominada Ley de Protección y Atención a los Migrantes en el Estado (Aquino, 2016). Uno de los puntos más controversiales en esta última ley mencionada fue la aprobación del artículo cuarto que establecía como derecho de los migrantes mostrar la documentación que acredite su identidad cuando le sea requerida por las autoridades competentes, así como proporcionar tanto información como datos personales que las instituciones del estado le soliciten. El argumento esgrimido por quien realizó la propuesta fue precisamente poder generar mecanismos de identificación, control y requisito para que los servicios del estado pudieran ser accesibles a las personas migrantes.

De manera interesante, fue la propia Comisión Nacional de Derechos Humanos (CNDH) quien a un mes después de la aprobación de dicha ley en octubre de 2017, se promulgó por una acción de inconstitucionalidad porque el artículo señalado violaba los derechos de la población migrante, de manera puntual los referidos a la libertad de tránsito, la integridad personal, la seguridad personal, la intimidad y la protección de datos personales.

La percepción de ilegalidad, vinculada a la necesidad de vigilar y controlar mediante diferentes instrumentos incrementa la vulnerabilidad de las personas migrantes en situación irregular que se encuentran en la ciudad. Para el alcalde de Guadalajara en funciones en ese momento (2012-2015), el estatus legal de los migrantes es una preocupación fundamental: "Porque el generar una serie de programas oficiales es tanto como aceptar o alentar el fenómeno de la migración" (alcande de Guadalajara, 2014). De esta manera el carácter racista del Estado se manifiesta en la identificación de una amenaza externa que no debe ser fomentada, o que debe de ser contenida. 
El carácter racista del Estado también se evidencia en la caracterización que las autoridades locales hacen de los migrantes en tránsito, no solo como una amenaza de seguridad, sino como una de salud. En marzo de 2013, una regidora del Ayuntamiento de Zapopan ${ }^{10}$ presentó una iniciativa para que se abriera un albergue para migrantes en el municipio. De acuerdo con la propuesta presentada en el pleno del ayuntamiento, el objetivo del albergue sería el de brindar asistencia humanitaria a los migrantes en tránsito: un lugar para descansar, comer y tomar un baño. Sin embargo, en el cuerpo de la iniciativa se hacía notar una preocupación adicional. De acuerdo con la regidora, cuando estaba elaborando la iniciativa, la Secretaría de Salud de Jalisco le advirtió que las personas migrantes en tránsito provenientes de Centroamérica representaban un foco de infección como portadoras de enfermedades que ya habían sido erradicadas en México, pero no en esos países. Las personas migrantes en tránsito se convirtieron entonces también en una amenaza biológica que debía ser debidamente contenida y controlada. Cuando se entrevistó a la regidora, señaló: "Necesitamos una casa en la cual sepamos cuáles están entrando, cuáles están saliendo, cuáles ya vacunamos, cuáles no. Y nosotros vemos el fondo de por la gente que está alrededor" (regidora de Ayuntamiento de Zapopan, 2014). Entonces tal como describía Foucault, el racismo del Estado opera creando una amenaza externa representada por los cuerpos de los migrantes en tránsito, cuerpos de los que los ciudadanos que viven en las áreas cercanas a las vías del tren tienen que ser protegidos. Es así como se dibuja una frontera simbólica, basada en una supuesta amenaza biológica, entre aquellos que pertenecen y los que no.

No son pocas las iniciativas gubernamentales en los municipios de Guadalajara y Zapopan para la construcción de albergues para migrantes en la ZMG. De hecho, la construcción de albergues han sido las únicas propuestas que los gobiernos municipales han presentado es relación con la migración en tránsito por la región. Los albergues resultan fundamentales para las autoridades locales porque les brindan la oportunidad de controlar y estabilizar los cuerpos de la población migrante en un lugar determinado. De fondo no importa realmente la necesidad de apoyo humanitario integral frente al abandono y riesgo al que se exponen las personas migrantes en tránsito por México, sino garantizar espacios que no rompan con los imaginarios sociales establecidos, y preservar a la ciudad de los cuerpos no autorizados.

Estas propuestas, como la de los albergues públicos para migrantes, son frágiles (ninguna se ha consolidado) dado que para las autoridades locales, la atención a los migrantes solo puede estar basada en un modelo asistencialista. Para el Alcalde de Guadalajara: "La participación de la autoridad municipal podría ser solo de carácter humanitario, y tiene que ver con el establecimiento de algunas medidas de apoyo a quienes están en esta situación, pero solo podrían ser asistencialistas" (alcalde de Guadalajara, 2014). Esta declaración pone en evidencia el carácter frágil, coyuntural y temporal de cualquier participación del gobierno local relacionado con la migración en tránsito.

A la fecha, ni las legislaciones estatales, ni los reglamentos municipales han emprendido las acciones necesarias para armonizar sus marcos normativos con lo establecido en la Ley de Migración 2011 y el Programa Especial de Migración de 2014-2018, tampoco han

\footnotetext{
${ }^{10} \mathrm{El}$ nombre de la regidora no se revela puesto que el informe consentido que firmó para la entrevista establecía que su nombre no sería utilizado y únicamente se mostraría el periodo de su administración que es 2012-2015.
} 
establecido programas o proyectos que de manera institucional puedan cumplir con lo mandatado en la Constitución. De acuerdo con el presidente municipal de Zapopan, “[...] pues no quisiera una llamarlo una desatención, pero sí una indiferencia tal vez del INM. Nunca la Federación ha bajado los programas a los municipios" (alcalde de Zapopan, 2014). Es precisamente a través de esta indiferencia institucional que las autoridades en todos sus niveles, ejerciendo el poder soberano al que se refiere Foucault, deciden ignorar a las personas migrantes como sujetos políticos, negándoles una existencia política y delineando una frontera simbólica entre los ciudadanos y los otros. Como consecuencia, las autoridades no asumen responsabilidad alguna sobre la migración en tránsito, lo que trabaja en favor de su invisibilización.

En ese sentido, las prácticas del Estado a nivel local han estado basadas no en la persecución de las personas migrantes o del establecimiento de mayores controles migratorios, sino en esfuerzos por categorizar, nombrar e identificar a la población migrante, por mantenerla y ubicarle en un espacio específico. Por tanto, las prácticas del Estado, su gubernamentalización se traduce en tácticas para hacerlos visibles, lo que de acuerdo con Tazzioli y Walters (2016), son técnicas para el control y detección de los movimientos de las personas migrantes. Es decir, a nivel local, el despliegue de las instituciones del Estado se ha centrado en visibilizar, categorizar y estabilizar a la población migrante con el objetivo de tener un control sobre la misma, además de poder -en su caso- eliminar las amenazas biológicas que se argumenta, podrían llegar a representar.

\section{Consideraciones finales}

Las dinámicas de la migración en tránsito por México, en este caso por la Zona Metropolitana de Guadalajara, ponen en evidencia la existencia de prácticas puntuales de refronterización del Estado que se visibilizan a través de la puesta en marcha de acciones y políticas como el Programa Integral Frontera Sur, y acuerdos económicomilitares como el Plan Mérida, así como a través de discursos institucionales que son asumidos e interiorizados por sujetos particulares, "ciudadanos", para marcar límites entre los cuerpos "autorizados" y "no autorizados"; entre sujetos de derecho y los que no lo son.

Dicha refronterización ha implicado diversos procesos políticos, institucionales, económicos y socioculturales, manifiestos también en la multiplicación y ampliación de las fronteras, un drástico aumento de programas que buscan regular y controlar el flujo migratorio a través de México, y un marcado incremento de operativos migratorios y de puestos de control fronterizo, hechos todos que han derivado en condiciones de mayor peligrosidad e invisibilidad para las personas migrantes en tránsito por México.

Los nuevos controles migratorios operan además diferenciando y segmentando a la población migrante en tránsito por la ZMG. Aunada a la política migratoria nacional - ligada a la política de Estados Unidos-, en las ciudades las prácticas de las autoridades actúan de manera desarticulada respondiendo a una lógica de control no para deportar o impedir que las personas migrantes lleguen a Estados Unidos, sino para primero, asegurarse que no permanecerán en la ciudad, y en segundo lugar, para poder categorizarlos, reconocerlos, y eliminar las amenazas biológicas que, desde su 
punto de vista, pudieran representar. Todo ello, deviene en la negación de su estatus como sujetos políticos, y por tanto sujetos de derechos. Estas prácticas marcan una clara línea entre los cuerpos autorizados a estar en la ciudad y a desplazarse por ella libremente y los cuerpos no autorizados, las personas migrantes en tránsito por la ZMG.

La consolidación de una doble imaginación geográfica designa tanto a los bienes que se transportan como al tren de carga como sujetos de protección, otorgándoles la libertad de moverse a lo largo del territorio nacional; mientras que a las personas migrantes las somete a una disciplina fronteriza rigurosa. De un lado los bienes se consolidan como símbolos del progreso, mientras que los migrantes en tránsito por el país se convierten en cuerpos que deben ser expulsados, controlados o desechados.

Lo anterior deriva en un marco en el que las acciones del Estado no son estables, coherentes o estáticas y donde diferentes imaginarios políticos, económicos y socioculturales devienen en prácticas contradictorias que se complejizan en un escenario como el de la ZMG. Es decir, las acciones del Estado reflejan una disociación entre las funciones de la frontera, y sus prácticas a través de ensanchamiento de las fronteras a territorios como la ZMG. Los múltiples esfuerzos intencionales por extender la frontera han orillado a las personas migrantes a la invisibilidad y a recorrer caminos y rutas cada vez más peligrosas.

Los procesos de refronterización del Estado aquí descritos se conjuntan para construir espacios en los que por un lado, la vulnerabilidad de las personas migrantes en tránsito se intensifica, y por el otro, revelan la fragilidad y porosidad de estas prácticas.

\section{Referencias}

Acharya, A. K. (2010). Feminization of migration and trafficking of women in Mexico. Review of Research and Social Intervention, 30, 19-38.

Aquino, M. (10 de junio de 2016). ‘Disputan’ diputados reforma migrante. El Norte. Recuperado de https://www.elnorte.com/aplicacioneslibre/preacceso/articulo/default.aspx?id=866126\&v=2\&flow_type=paywall\&urlredirect=https: / www.elnorte.com/aplicaciones/articulo/default.aspx?id=866126\&v=2\&flow_ type=paywall

Brenner, N. (2004). New state spaces: Urban governance and the rescaling of statehood. Oxford, Nueva York: Oxford University Press.

Brenner, N., Jessop, B., Jones, M. y MacLeod, G. (2008). State/Space: A Reader. Hoboken: Wiley.

Burridge, A., Nick, G., Austin, K. y Martin, L. (2017). Polymorphic borders. Territory, Politics, Governance, 5(3), 239-251. doi:10.1080/21622671.2017.1297253

Casillas, R. (2008). Las rutas de los centroamericanos por México, un ejercicio de caracterización, actores principales y complejidades. Migración y Desarrollo, (10), 157-174. Recuperado de http:/ / www.redalyc.org/articulo.oa?id=66001007

Chatterjee, P. (2004). The politics of the governed: Reflections on popular politics in the most of the world. Nueva York: Columbia University Press.

De Leon, M. L. (2013). Feminization of migration: A global health challenge. Global Advances in Health and Medicine, 2(5), 12-14. doi:10.7453/gahmj.2013.065 
Encuesta sobre Migración en la Frontera Sur (EMIF SUR). (2012). Encuesta sobre Migración en la Frontera Sur, 2009. INM, CONAPO, COLEF. SEGOB, SRE, STPS. México.

El Informador. (10 de marzo de 2016). Diputados de Jalisco buscan ampliar derechos a migrantes. Recuperado de https://www.informador.mx/Jalisco/Diputados-de-Jalisco-buscan-ampliar-derechos-a-migrantes-20160310-0066.html

Fassin, D. (2011). Policing borders, producing boundaries. The governmentality of immigration in dark times. Annual Review of Anthropology, 40, 213-226.

FM4 Paso Libre. (2013). Migración en tránsito por la Zona Metropolitana de Guadalajara: Actores, retos y perspectivas desde la experiencia de FM4 Paso Libre. Guadalajara, México: FM4 Paso Libre.

Foucault, M. ([1976] 2003). Society must be defended: Lectures at the Collège de France, 197576. Nueva York: Picador.

García, M. del C. y Villafuerte, D. (2014). Migración, derechos humanos y desarrollo, aproximaciones desde el sur de México y Centroamérica. México: unicach, Juan Pablos Editor.

Heyman, J. McC y Ackleson, J. (2009). United States border security after September 11. En J. Winterdyck y K. Sundberg (Eds.), Border Security in the Al-Qaeda Era (pp. 37-74). Boca Raton, Florida: CRC Press.

Massey, D. (2005). For Space. Londres; Thousand Oaks, California: SAGE.

REDODEM. (2015). Migrantes invisibles, violencia tangible. Informe 2014. México: Red de Documentación de Defensores de Migrantes.

Ribando, C. y Finklea, K. (2017). U.S.-Mexican Security Cooperation: The Mérida Initiative and Beyond. Congressional Research Service. Recuperado de https://fas.org/ $\mathrm{sgp} / \mathrm{crs} / \mathrm{row} / \mathrm{R} 41349 . \mathrm{pdf}$

Tazzioli, M. y Walters, W. (2016). The sight of migration: Governmentality, visibility and Europe's contested borders. Global Society, 30(3), 445-464. doi: $10.1080 / 13600826.2016 .1173018$

Toney, J. (2009). The caribbean and the feminization of emigration: Effects and repercussions. Wadabagei: A Journal of the Caribbean and Its Diaspora, 12(1), 60-72.

Torre-Cantalapiedra, E. y Yee-Quintero, J. C. (2018). México ¿una frontera vertical? Políticas de control del tránsito migratorio irregular y sus resultados, 20072016. LiminaR, 16(2), 87-104.

Alcalde de Guadalajara. (Verano de 2014). Entrevista realizada al alcalde de Zapopan durante la administración 2012-2014. Entrevistador [Entrevista realizada por uno de los autores durante el verano (meses) de 2014]. Lugar.

Alcalde de Zapopan. (Verano de 2014). Entrevista realizada al alcalde de Zapopan durante la administración 2012-2014. Entrevistador [Entrevista realizada por uno de los autores durante el verano (meses) de 2014]. Lugar.

Habitante de El Verde. (2014). Entrevista realizada por los autores. Lugar.

Regidora del Zapopan. (2014). Regidora de Zapopan durante la administración 20122014. Entrevista realizada por uno de los autores durante el verano de 2014. 


\section{Entrevistas}

Alcalde de Guadalajara. (Verano de 2014). Por autores. Entrevista realizada al alcalde de Guadalajara durante la administración 2012-2015. Jalisco, México.

Alcalde de Zapopan. (Verano de 2014). Por autores. Entrevista realizada al alcalde de Zapopan durante la administración 2012-2015. Jalisco, México.

Habitante de El Verde. (13 de junio de 2014). Por Mariana Morante Aguirre. El Salto, Jalisco.

Mayordomo. (Verano de 2014). Por autores. Jalisco, México.

Regidora de Zapopan. (Verano de 2014). Por autores. Entrevista realizada a regidora de Zapopan durante la administración 2012-2015. Jalisco, México.

Rafael Alonso Hernández López

Mexicano. Doctor en Ciencias Sociales y Humanísticas por CESMECA-Unicach. Coordinador del Doctorado en Estudios de Migración en El Colegio de la Frontera Norte, sede Tijuana. Miembro del sni nivel I. Presidente del Consejo Ciudadano del Instituto Nacional de Migración. Líneas de investigación: migración internacional en tránsito, solicitantes de refugio y refugiados, jornaleros migrantes y derechos humanos en la migración. Publicación reciente: Anguiano, M. E., Hernández, R. A. y Villafuerte, D. (2018). The world through borders: The difficult journey or migrants in transit. México: El Colegio de la Frontera Norte, Universidad de Ciencias y Artes de Chiapas, cesmeca.

Iván Francisco Porraz Gómez

Mexicano. Doctor en Ciencias Sociales y Humanísticas por CESMECA-UnICACH. Investigador asociado en El Colegio de la Frontera Sur, unidad Tapachula, en el Grupo Académico de Estudios de Migración y Procesos Transfronterizos del Departamento de Sociedad y Cultura. Miembro del SNI nivel candidato. Líneas de investigación: juventudes, migración de retorno, violencia en el sur de México y Centroamérica. Publicación reciente: García, M. del C., Porraz, I. F. (2018). Fenomenología de la violencia del siglo xxi en El Salvador: la juventud sin lugar. Anuario de Estudios Centroamericanos, Universidad de Costa Rica, (44), 341-366.

Mariana Morante Aguirre

Mexicana. Maestra en Estudios Latinoamericanos y Maestra en Política Global, ambas por la Universidad de Texas en Austin. Asistente de investigación de Dignidad y Justicia en el Camino. A.C. (FM4 Paso Libre). Líneas de investigación: Tránsito de personas migrantes indocumentadas por áreas urbanas; prácticas espaciales por la ciudad e imaginarios socioculturales. Políticas migratorias y sus efectos en el tránsito de las personas migrantes indocumentadas. Acceso a la justicia, justicia abierta y políticas de transparencia en México. Publicación reciente. Delgado, A. y Morales, M. (2018). Tensiones entre seguridad nacional y acceso a la información pública; un recuentro del recurso de revisión en materia de seguridad nacional ante la Suprema Corte de Justicia de la Nación, Revista del Centro de Estudios Constitucionales, (6), 295-340. 